\title{
Comparison Between Safety and Efficacy of Two Treatment Regimens for Pediatric Patients with Chronic Hepatitis C Virus Infection: Sofosbuvir/Ledipasvir Versus Sofosbuvir/Daclatasvir Regimen
}

\author{
Abdelfattah Hanno', Soraya Hamouda', Manal Abdelgawad ${ }^{2}$, Hossam Aboelkheir ${ }^{1 *}$, Mohamed Ahmed ${ }^{1}$ \\ ${ }^{1}$ Tropical Medicine Department, Faculty of Medicine, Alexandria University, Egypt \\ ${ }^{2}$ Pediatric Department, Faculty of Medicine, Alexandria University, Egypt \\ *Corresponding Author: Hossam Aboelkheir, Tropical Medicine Department, Faculty of Medicine, Alexandria University, Egypt.
}

Received: June 07, 2019; Published: August 07, 2019

\begin{abstract}
Background: It is estimated that 11 million children under the age of 15 are infected by Hepatitis C virus (HCV), of whom 5 million are viremic. In the Egyptian children population (1- 14 years old), the prevalence of HCV antibody and HCV RNA are estimated to be $0.4 \%$ and $0.2 \%$ respectively, with genotype 4 being the most prevalent. The treatment regimen that is currently approved for children is a combination therapy of Ledipasvir/sofosbuvir (LDV/SOF) for genotype1,4,5 and 6, while genotype 2 or 3 can be treated cautiously with regimens approved for adults.
\end{abstract}

Objective: The aim of this study is to compare the efficacy and safety of sofosbuvir/ledipasvir versus sofosbuvir/daclatasvir.

Patients and Methods: Forty treatment naïve and experienced patients aged 10 to 17 years with PCR confirmed chronic HCV infection were enrolled and randomly assigned to two groups. Both groups were matched to age, sex and BMI. Patients in group I received LDV/SOF 90mg/400mg once daily for 12 weeks while group II received DCV/SOF 60mg/400mg once daily for the same period. For patients in group I under $35 \mathrm{~kg}$, and under $40 \mathrm{~kg}$ in group II, half the mentioned dose was given. Efficacy (in terms of a sustained virologic response-SVR) was assessed using PCR for HVC at week 4, week 12 (end of treatment) and 12 weeks after end of treatment (SVR 12) while safety was evaluated using close clinical follow-up and weekly laboratory investigations at three different intervals; weekly during the first month of treatment, monthly until end of treatment, and at 12 weeks from the last dose.

Patients and Methods: Forty treatment naïve and experienced patients aged 10 to 17 years with PCR confirmed chronic HCV infection were enrolled and randomly assigned to two groups. Both groups were matched to age, sex and BMI. Patients in group I received LDV/SOF 90mg/400mg once daily for 12 weeks while group II received DCV/SOF 60mg/400mg once daily for the same period. For patients in group I under $35 \mathrm{~kg}$, and under $40 \mathrm{~kg}$ in group II, half the mentioned dose was given. Efficacy (in terms of a sustained virologic response-SVR) was assessed using PCR for HVC at week 4, week 12 (end of treatment) and 12 weeks after end of treatment (SVR 12) while safety was evaluated using close clinical follow-up and weekly laboratory investigations at three different intervals; weekly during the first month of treatment, monthly until end of treatment, and at 12 weeks from the last dose.

Results: All patient ( $\mathrm{n}=40)$ in our study achieved SVR 12 (100\%). A significant decrease in ALT and AST was observed. No major side effects necessitating termination of treatment were observed in either group. The most common side effects were abdominal pain (40\%) and fatigue (40\%) in group I, and headache (45\%) and abdominal pain (35\%) in group II.

Conclusion: A 12-week regimen of LDV/SOF and DCV/SOF appear to achieve the same results in regard to efficacy and safety in the young population.

Keywords: HCV; Ledipasvir; Sofosbuvir; Daclatasvir

\section{Introduction}

The total global prevalence of anti-HCV was estimated to be $1.6 \%(1.3-2.1 \%)$, corresponding to 115 (92-149) million past viremia infections. The majority of these infections, (87-124) million, were among adults (defined as those older than 15 years old) with an anti-HCV infection rate of $2.0 \%(1.7-2.3 \%)$ [1].

The ratio of HCV prevalence among children to adults was 54\% in low-income countries, $28 \%$ in lower-middle-income countries,
$21 \%$ in upper-middle-income countries and $4 \%$ in high-income countries. Given the high uncertainty associated with this approach, a range of $4-54 \%$ was used for all regions. An average viraemic rate of $50 \%$ (uncertainty interval of 50-75\%) was applied to the infected population aged $<15$ years $[2-4]$.

HCV still affects a substantial proportion of the Egyptian population, where it is estimated that, in the 1-59-year age group, 5.3 million persons are positive for HCV antibodies and, of these, ap- 
proximately 3.7 million (69.5\%) are HCV RNA positive. This is an underestimate of the total human HCV reservoir in Egypt because older age groups ( $>59$ years) were not included in the EHIS (Egyptian health issue survey) 2015 [5].

In Egyptian children, 1-14 years old, the prevalence of HCV antibody and HCV RNA were $0.4 \%$ (95\% CI 0.3-0.5) and 0.2\% (95\% CI 0.1-0.3) respectively [5].

In April 2017, the European Medicines Agency approved the fixed-dose combination of sofosbuvir and ledipasvir (for genotypes $1,4,5$ and 6) and the combination of sofosbuvir and ribavirin (for genotypes 2 and 3) for adolescents aged 12-17 years, or weighing greater than $35 \mathrm{~kg}$, with chronic hepatitis C. Thus, IFN-based treatment is no longer preferred [6].

\section{Aim of the work}

The aim of this study is to assess the efficacy and safety of combined Sofosbuvir/Ledipasvir and Sofosbuvir/Daclatasvir for treatment of childre Results $n$ aged 10-17 with chronic hepatitis $C$ virus.

\section{Patients and Methods}

Forty patients whom their age ranged from 10-17 years with chronic hepatitis C virus recruited from Hepatology clinic, EL-Shatby pediatric hospital, Faculty of Medicine, Alexandria University and divided into 2 equal groups.

- 20 patients age $10-17$ years with chronic hepatitis $C$ virus treated with Sofosbuvir (400mg)/Ledipasvir (90mg) once daily for 12 weeks

- 20 patient age 10-17 years with chronic hepatitis $C$ virus treated with Sofosbuvir (400mg)/Daclatasvir (60mg) once daily for 12 weeks.

\section{Exclusion criteria}

Patients with concurrent co-infection with hepatitis B, diabetes mellitus, those suffering from fever, patients with autoimmune hepatitis and cirrhotic patients

\section{Ethical approval}

The ethical committee of Faculty of Medicine, Alexandria University, approved this study. Written informed consent was obtained from all patient's parents.

Methods: All patients who tested HCV antibody positive were subjected to:

- Detailed history taking and thorough clinical examination.

- Laboratory investigations:

\section{Routine investigations}

CBC, blood urea, serum creatinine, aspartate transaminase, alanine transaminase, total and direct bilirubin, prothrombin
- Activity, INR, total protein, and serum albumin will be done weekly for the first month and then monthly till end of treatment

- HCV RNA PCR (polymerase chain reaction) before start of treatment, at the 4 th week of treatment and at the end of treatment and 3 months after end of treatment

- HBsAg before beginning of treatment

- ANA and anti LKMA before beginning of treatment

- Alpha-feto protein before and after treatment

- ECG before start of treatment to exclude any arrhythmias

Body mass index (BMI) was calculated at the beginning of HCV treatment and after 6 months of treatment by the following formula: $\mathrm{BMI}=$ weight in $\mathrm{Kg} /{\text { (height in meter })^{2}}^{2}$

\section{Radiological studies}

- Ultrasound study of the abdomen and pelvis before start and after finishing treatment

- Fibroscan before start of treatment

\section{Results}

Demographic data

\begin{tabular}{|c|c|c|c|c|c|c|}
\hline & \multicolumn{2}{|c|}{$\begin{array}{l}\text { Group I } \\
(n=20)\end{array}$} & \multicolumn{2}{|c|}{$\begin{array}{c}\text { Group II } \\
(\mathbf{n}=20)\end{array}$} & \multirow{2}{*}{$\begin{array}{c}\text { Test of } \\
\text { sig. }\end{array}$} & \multirow[t]{2}{*}{$\mathbf{P}$} \\
\hline & No. & $\%$ & No. & $\%$ & & \\
\hline \multicolumn{7}{|l|}{ Sex } \\
\hline Male & 12 & 60.0 & 11 & 55.0 & \multirow[t]{2}{*}{$c^{2}=0.102$} & \multirow[t]{2}{*}{0.749} \\
\hline Female & 8 & 40.0 & 9 & 45.0 & & \\
\hline \multicolumn{7}{|l|}{ Age (years) } \\
\hline Min. - Max. & \multicolumn{2}{|c|}{$10.0-17.0$} & \multicolumn{2}{|c|}{$10.0-17.0$} & \multirow[t]{3}{*}{$\mathrm{t}=1.061$} & \multirow[t]{3}{*}{0.296} \\
\hline Mean \pm SD & \multicolumn{2}{|c|}{$14.05 \pm 2.04$} & \multicolumn{2}{|c|}{$13.35 \pm 2.13$} & & \\
\hline Median & \multicolumn{2}{|c|}{14.0} & \multicolumn{2}{|c|}{13.50} & & \\
\hline \multicolumn{7}{|c|}{ BMI $\left(\mathrm{kg} / \mathrm{m}^{2}\right)$} \\
\hline Min. - Max. & \multicolumn{2}{|c|}{$17.98-32.28$} & \multicolumn{2}{|c|}{$15.92-32.87$} & \multirow[t]{3}{*}{$\mathrm{t}=0.590$} & \multirow[t]{3}{*}{0.559} \\
\hline Mean \pm SD & \multicolumn{2}{|c|}{$22.82 \pm 3.36$} & \multicolumn{2}{|c|}{$22.05 \pm 4.79$} & & \\
\hline Median & \multicolumn{2}{|c|}{22.72} & \multicolumn{2}{|c|}{21.99} & & \\
\hline
\end{tabular}

Table 1: Comparison between the two studied groups according to demographic data.

$\chi^{2}$ : Chi square test

t: Student t-test

$\mathrm{p}$ : $\mathrm{p}$ value for comparing between the two groups

*: Statistically significant at $\mathrm{p} \leq 0.05$

\section{Previous treatment}

Previous treatment consisted of interferon, in group I, 11 patients (55\%) had no previous treatment while 9 patients (45\%) were previously treated with interferon. On the other hand, 13 patients (65\%) of group II had no previous exposure to treatment while 7 patients (35\%) were treated with interferon. There was no statistically significant difference between the two groups. 
Hemoglobin level (Hb)(gm/dl)

There was no significant difference between the hemoglobin level of the 2 groups.

\begin{tabular}{|c|c|c|c|c|}
\hline & $\begin{array}{l}\text { Group I } \\
(n=20)\end{array}$ & $\begin{array}{c}\text { Group II } \\
(n=20)\end{array}$ & $\mathbf{T}$ & $\mathbf{P}$ \\
\hline \multicolumn{5}{|l|}{ Week 0} \\
\hline Min. - Max. & $11.30-15.50$ & $11.0-14.80$ & \multirow[t]{3}{*}{1.326} & \multirow[t]{3}{*}{0.193} \\
\hline Mean \pm SD & $13.84 \pm 1.15$ & $13.35 \pm 1.19$ & & \\
\hline Median & 13.80 & 13.80 & & \\
\hline \multicolumn{5}{|l|}{ Week 12} \\
\hline Min. - Max. & $11.0-15.40$ & $1.50-15.20$ & \multirow[t]{3}{*}{1.858} & \multirow[t]{3}{*}{0.071} \\
\hline Mean \pm SD & $13.64 \pm 1.09$ & $12.40 \pm 2.78$ & & \\
\hline Median & 13.65 & 12.50 & & \\
\hline \multicolumn{5}{|l|}{ Week 24} \\
\hline Min. - Max. & $11.40-15.40$ & $11.90-15.10$ & \multirow[t]{3}{*}{ ds1.454 } & \multirow[t]{3}{*}{0.154} \\
\hline Mean \pm SD. & $13.66 \pm 1.0$ & $13.21 \pm 0.98$ & & \\
\hline Median & 13.55 & 13.10 & & \\
\hline
\end{tabular}

Table 2: Comparison between the two studied groups according to $\mathrm{Hb}$.

t: Student t-test

White blood count (thousands/ul)

As regarding the white blood count, there was no significant difference between the WBC between the two groups at week 0, 4 and 8 while there was a difference at week 12 and 24.

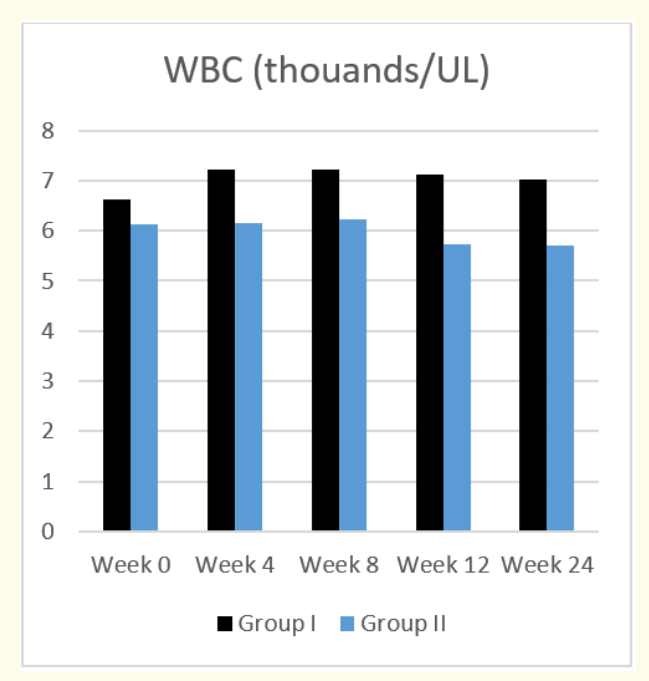

Figure 1: Comparison between 2 groups regarding WBC level at different treatment periods.

Both groups showed no significant difference as regarding the WBC through the treatment period.
Absolute neutrophilic count (ANC)(thousands/UL)

There was statistically significant difference between the two groups at week 12 and 24 .

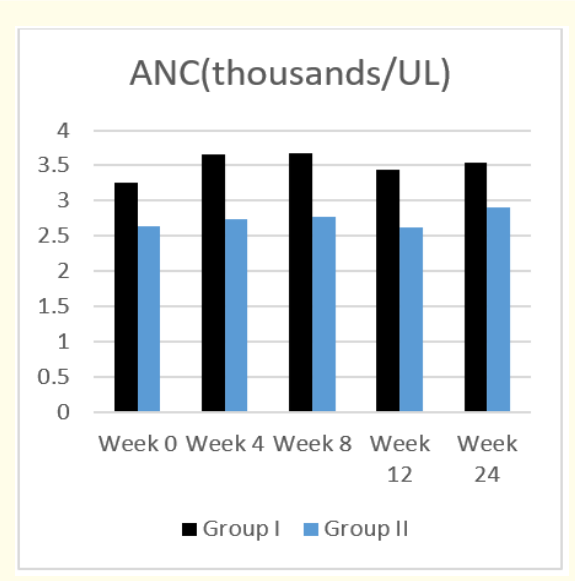

Figure 2: Comparison between 2 groups regarding ANC level at different treatment periods.

Liver enzymes ( $\mathrm{u} / \mathrm{l})$

There was statistically significant difference in both groups across the treatment period regarding AST and ALT.

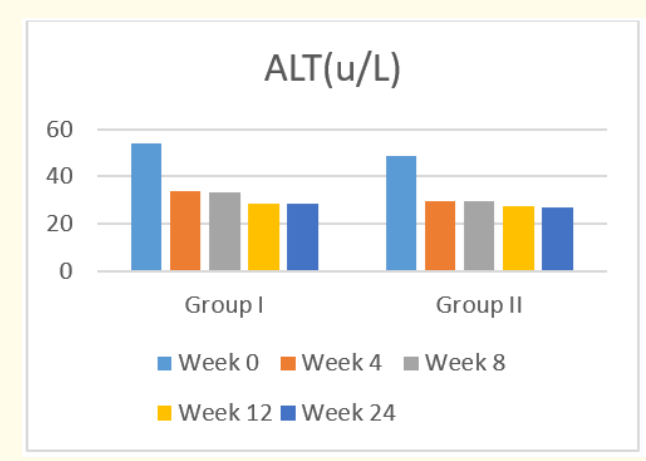

Figure 3: Comparison for each group regarding ALT level at different treatment periods.

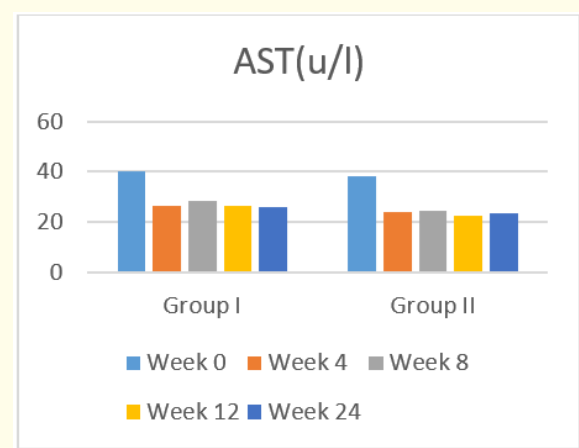

Figure 4: Comparison for each group regarding AST level at different treatment periods 


\section{PCR during treatment}

$100 \%$ of patients of both group I and II has PCR $<15$ at weeks 4 , 12 and 24 .

\begin{tabular}{|l|c|c|c|c|c|c|c|c|}
\hline \multirow{2}{*}{ PCR } & \multicolumn{2}{|c|}{$\mathbf{0}$} & \multicolumn{2}{c|}{$\mathbf{4}$} & \multicolumn{2}{c|}{12} & \multicolumn{2}{c|}{24} \\
\cline { 2 - 9 } & No. & $\%$ & No. & $\%$ & No. & $\%$ & No. & $\%$ \\
\hline $\begin{array}{l}\text { Group I } \\
\text { (n=20) }\end{array}$ & & & & & & & & \\
\hline$<15$ & 0 & 0.0 & 20 & 100.0 & 20 & 100.0 & 20 & 100.0 \\
\hline$>15$ & 20 & 100.0 & 0 & .0 & 0 & 0.0 & 0 & 0.0 \\
\hline $\begin{array}{l}\text { Group II } \\
\text { (n=20) }\end{array}$ & & & & & & & & \\
\hline$<15$ & 0 & 0.0 & 20 & 100.0 & 20 & 100.0 & 20 & 100.0 \\
\hline$>15$ & 20 & 100.0 & 0 & 0.0 & 0 & 0.0 & 0 & 0.0 \\
\hline
\end{tabular}

Table 4: Comparison between the different periods according to PCR.

\section{Side-effects}

There were no major side effects necessitating termination of treatment in both groups. The most common side effects in group I were abdominal pain and fatigue while in group II was headache.

\begin{tabular}{|c|c|c|c|c|c|c|}
\hline & \multicolumn{2}{|c|}{$\begin{array}{l}\text { Group I } \\
(n=20)\end{array}$} & \multicolumn{2}{|c|}{$\begin{array}{c}\text { Group II } \\
(n=20)\end{array}$} & \multirow[t]{2}{*}{$c^{2}$} & \multirow[t]{2}{*}{$\mathbf{P}$} \\
\hline & No. & $\%$ & No. & $\%$ & & \\
\hline \multicolumn{7}{|c|}{ Abdominal pain } \\
\hline None & 12 & 60.0 & 13 & 65.0 & \multirow[t]{2}{*}{0.107} & \multirow[t]{2}{*}{0.744} \\
\hline Mild & 8 & 40.0 & 7 & 35.0 & & \\
\hline \multicolumn{7}{|c|}{ Headache } \\
\hline None & 15 & 75.0 & 12 & 60.0 & \multirow[t]{2}{*}{1.026} & \multirow[t]{2}{*}{0.311} \\
\hline Mild & 5 & 25.0 & 8 & 40.0 & & \\
\hline \multicolumn{7}{|c|}{ Diarrhea } \\
\hline None & 17 & 85.0 & 20 & 100.0 & \multirow[t]{2}{*}{3.243} & \multirow[t]{2}{*}{0.231} \\
\hline Mild & 3 & 15.0 & 0 & 0.0 & & \\
\hline \multicolumn{7}{|c|}{ Vomiting } \\
\hline None & 18 & 90.0 & 13 & 65.0 & \multirow[t]{2}{*}{3.584} & \multirow[t]{2}{*}{0.127} \\
\hline Mild & 2 & 10.0 & 7 & 35.0 & & \\
\hline \multicolumn{7}{|c|}{ Nausea } \\
\hline None & 17 & 85.0 & 14 & 70.0 & \multirow[t]{2}{*}{1.290} & \multirow[t]{2}{*}{0.451} \\
\hline Mild & 3 & 15.0 & 6 & 30.0 & & \\
\hline \multicolumn{7}{|c|}{ Fatigue } \\
\hline None & 12 & 60.0 & 16 & 80.0 & \multirow[t]{2}{*}{1.905} & \multirow[t]{2}{*}{0.168} \\
\hline Mild & 8 & 40.0 & 4 & 20.0 & & \\
\hline \multicolumn{7}{|c|}{ Pruritis } \\
\hline None & 15 & 75.0 & 14 & 70.0 & \multirow[t]{2}{*}{0.125} & \multirow[t]{2}{*}{0.723} \\
\hline Mild & 5 & 25.0 & 6 & 30.0 & & \\
\hline
\end{tabular}

Table 5: Comparison between the two studied groups according to different parameters.

\section{Discussion}

The pre-treatment parameters of our subjects was in concordance with Balistreri., et al. [7] with hemoglobin over 11g/dl. There was no significant drop across the treatment period in both groups.

The pre-treatment absolute neutrophil count was similar in group I with Balistreri., et al. [7] lower cut point of 1500cells/ul and 600cells/ul for group II. Although some patients in group II had lower ANC at the beginning of treatment, there were no statistically significant difference across the treatment period which is in agreement with Yakoot., et al. [8] and El-Sayed., et al. [9] whom reported no significant adverse effect regarding the ANC.

Group II had lower white blood count and absolute neutrophilic than group I at week 12 and 24. This finding was difficult to justify due to absence of head to head trials comparing both treatment regimens and further studies is needed to investigate these findings, however it should be noted that each group separately did not show any significant difference across the treatment period regarding WBC and ANC.

Both groups showed significant decrease in ALT level across the treatment period and in the 12 week follow-up after the end of treatment which is in agreement with Balistreri., et al [7], Yakoot., et al. [8] and El-Sayed., et al [9].

Both groups showed significant decrease in AST level across the treatment period and in the 12 week follow-up after the end of treatment which is in agreement with Balistreri., et al. [7], Yakoot., et al [8], El-Sayed., et al. [9] and El-Khayat., et al [10].

PCR level showed no significant difference between the two groups at the start of therapy and all subjects in both groups achieved negative PCR at week 4 and remained negative till three months after end of treatment. There were no significance of the pre-treatment PCR level and response to treatment.

Balisteri., et al. [7] achieved a 98\% SVR 12 in their study, this slight difference was due to loss of follow-up, however our results were consistent with the mentioned results. The situation was similar with Yakoot., et al. [8] who also lost one patient to follow-up and achieved a SVR 12 of $96.7 \%$. El-Khayat., et al. [10] also achieved 99\% while El-Sayed., et al. [9] and El-Shabrawi., et al. [11] achieved SVR 12 of $100 \%$.

Studies with larger number of subjects tend to have loss of follow-up while studies with small number of subjects had no losses of patients which highlights the role of follow-up in ensuring adherence of patients to therapy.

The most reported side effect in group I was abdominal pain and fatigue $40 \%$, while Balistreri., et al. [7] reported only $7 \%$ of patients had abdominal pain. This may be due to higher incidence of para- 
sitic infestation in Egypt. On the other hand, the most reported side effect in group II was headache and that was similar to El-Sayed., et al. [9] who also reported headache as a common side effect in a Daclatasvir/Sofosbuvir treated group. It should be noted that these side-effects did not necessitate withdrawal of treatment in both groups.

EL-Khayat., et al. [10] reported that headache was the most common side effect in Ledipasvir/Sofosbuvir treated group which is similar to Balistreri., et al [7]. It should be noted that the mentioned two studies recruited larger number of patients which might account for the difference in the reported side-effects.

There were several strengths in our study, first of all the strict pre-treatment exclusion criteria allowed for accurate selection of patients and precise interpretation of outcomes. Besides, the fibroscan performed on all patients before treatment facilitated exclusion of patients with significant fibrosis or cirrhosis.

Moreover, the inclusion of treatment naïve and treatment experienced patients permitted us to evaluate the effect of previous exposure to interferon alpha on the response to the current treatment regimens implemented in our study.

In addition, the close monitoring of our patients during treatment and after that allowed us to ensure compliance to treatment and also monitor for adverse side effects either clinical or laboratory.

On the other hand, there were some limitations in our study, the most important one is the limited size of both groups which could have limited the ability to recognize some of less common side effects of both treatment regimens.

Another limitation is absence of follow-up fibroscan which could have helped us document resolution of liver inflammation.

\section{Bibliography}

1. Gower E., et al. "Global epidemiology and genotype distribution of the hepatitis C virus infection". Journal of Hepatology 61.1 (2014): S45-S57.

2. Seeff LB. "The history of the "natural history" of hepatitis C (1968-2009)". Liver International 29 (2009): 89-99.

3. Alter MJ., et al. "The prevalence of hepatitis $\mathrm{C}$ virus infection in the United States, 1988 through 1994". New England journal of medicine 341.8 (1999): 556-562.

4. Barakat $\mathrm{SH}$ and El-Bashir N. "Hepatitis C virus infection among healthy Egyptian children: prevalence and risk factors". Journal of viral hepatitis 18.11 (2011): 779-784.
5. Kandeel A., et al. "The prevalence of hepatitis C virus infection in Egypt 2015: implications for future policy on prevention and treatment". Liver International 37 (2017): 45-53.

6. Balistreri WF., et al. "The safety and effectiveness of ledipasvir-sofosbuvir in adolescents 12-17 years old with hepatitis C virus genotype 1 infection. Hepatology 66.2 (2017): 371-378.

7. Balistreri WF., et al. "The safety and effectiveness of ledipasvir- sofosbuvir in adolescents 12-17 years old with hepatitis C virus genotype 1 infection". Hepatology 66 (2017): 371-378.

8. Yakoot M., et al. "Dual sofosbuvir/daclatasvir therapy in adolescent patients with chronic hepatitis C infection". Journal of pediatric gastroenterology and nutrition 67 (2018): 86-89.

9. El-Sayed M., et al. "A pilot study for safety and efficacy of 12 weeks sofosbuvir plus daclatasvir with or without ribavirin in Egyptian adolescents with chronic hepatitis C virus Infection". Journal of Hepatology 66 (2017): S178.

10. El-Khayat H., et al. "The effectiveness and safety of ledipasvir plus sofosbuvir in adolescents with chronic hepatitis $\mathrm{C}$ virus genotype 4 infection: a real-world experience". Alimentary pharmacology and therapeutics 47 (2018): 838-844.

11. El-Shabrawi MH., et al. "Shortened 8 Weeks Course of Dual Sofosbuvir/Daclatasvir Therapy in Adolescent Patients, With Chronic Hepatitis C Infection". Journal of Pediatric Gastroenterology and Nutrition 66 (2018): 425-427.

\section{Volume 2 Issue 7 September 2019}

(C) All rights are reserved by Hossam Aboelkheir., et al. 